\title{
Symptoms of stress predict musculoskeletal disorders
}

\author{
PÄIVI LEINO \\ From the Institute of Public Health, University of Tampere, Box 607, SF-33101 Tampere, Finland.
}

\begin{abstract}
Study objective: To determine whether there is a relationship between musculoskeletal disorders and presumed symptoms of stress.

Design: Cross sectional and mixed longitudinal cohort study. Longitudinal data were collected at baseline (1973) and at re-examinations in 1978 and 1983.

Setting: Community based.

Participants: Study sample was drawn from employees who had worked for at least 15 months at government owned Valmet metal factories in Finland, and comprised 902 men and women out of a total eligible population of $2653 ; 74 \%$ of the women and $63 \%$ of the men took part in both re-examinations.

Measurements and main results: Musculoskeletal disorders were measured as rheumatic symptoms, clinical findings and presence of chronic specific diseases. Eighteen symptoms of stress were combined in a stress symptoms score. Stress symptoms were associated with rheumatic symptoms and clinical findings in all sex/occupational class groups at first examination (baseline); and the prevalence of chronic musculoskeletal disease was associated with stress symptoms in men and in blue collar women (skilled and semiskilled workers). The mean stress symptom score of 1973 and 1978 predicted the level of rheumatic symptoms and clinical findings in 1983, allowing for the relevant score at baseline. The mean score also predicted the incidence of disease during the second half of the follow up in women and in blue collar men. Rheumatic symptoms covaried with the stress symptoms. Change in stress symptoms was predicted by indices of musculoskeletal disorders.

Conclusions: The results suggest that stress symptoms and musculoskeletal disorders are reciprocally related.
\end{abstract}

Psychosocial stress, whether measured as life events or as more continuous strains, has received attention as a possible aetiological factor in musculoskeletal disorders. Consistent results have been obtained in support of a relationship between stressful life events and the onset of rheumatoid arthritis. ${ }^{1}$ Lichtenberg $e t$ $a l^{2}$ showed that stressful life events were associated with perceived pain in elderly patients with osteoarthritis, whereas arthritic severity was not. Minor events, called daily hassles, were shown by the same authors ${ }^{3}$ to be associated with arthritic pain, accounting for $22 \%$ of pain variance. In some studies contradictory or ambiguous results have been obtained. ${ }^{45}$

Aro $^{6}$ has observed that presumed symptoms of stress predict chronic diseases. The purpose of this paper was to study whether these symptoms are associated with the occurrence of musculoskeletal disorders in an industrial population.

The concept of stress was understood in the sense of an unbalanced relation between individual and environment: incongruity between personal needs/ goals and the resources available for their satisfaction/ attainment, or incongruity between environmental demands/loads and the capabilities/susceptibilities of the individual. ${ }^{67}$ Stress in the individual was assumed to be manifested as mental and somatic symptoms.

Musculoskeletal disorders were measured as rheumatic complaints, clinical findings made by a physiotherapist, and long term diseases reported by the subjects and checked by a physician. Both a cross sectional and a longitudinal study design was used. Two alternatives hypotheses of causation were tested: first, that stress predicts musculoskeletal disorders, and second, that musculoskeletal disorders produce stress. 
Methods

\section{THE STUDY SAMPLE}

The study sample was drawn from the employees ( $n=4570$ in 1973) of the government owned Valmet metal factories in Jyväskylä, central Finland. Data were gathered in the years 1973, 1978 and 1983 by a questionnaire, by interviews, and by a clinical examination of the musculoskeletal apparatus. The study population included those who had been employed by Valmet for at least 15 months at the beginning of the study $(n=2653)$. For the sampling the employees were stratified into groups according to sex, age (three groups: born in 1925 or earlier, born between 1926 and 1945, and born in 1946 or later) and occupational class (four groups: managers, other office staff, skilled workers, and semiskilled workers). The sample consisted of 902 persons in 1973. Skilled and semiskilled workers are pooled into one group (blue collar), and managers and office staff into another (white collar).

\section{DATA COLLECTION}

Preliminary information on the health status of the examinees was collected by a questionnaire, which was mailed to the members of the sample at the end of January each year. The questionnaire was completed in interviews in a health examination, during which an assessment of the clinical status of the musculoskeletal system was also made by one of two physiotherapists trained for the purpose. The health examinations took place during spring, from February to June.

\section{Symptoms of stress}

In the questionnaire, the subjects were asked: "Have you had some of the following symptoms during last year and how often? (Symptoms regularly associated with menstruation or with alcohol consumption should not be included)". These symptoms were selected from various treatises on mental stress and are generally assumed to be common in people undergoing stressful life periods. The following list of symptoms was given:

Heartburn or acid troubles
Loss of appetite
Nausea or vomiting
Abdominal pains
Diarrhoea or irregular bowel
function
Difficulties in falling asleep
or awakening during the
night
Nightmares
Headache
Sexual unwillingness

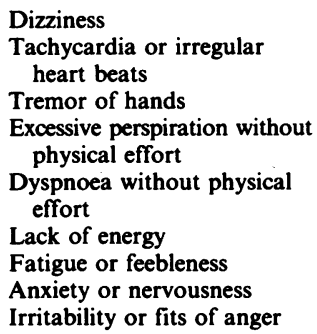

Each item was scored from 1 (seldom or never) to 4 (often or continuously). A sum score, labelled the stress symptoms score (SSS), was constructed. For the longitudinal analyses, a mean stress symptoms score was calculated from the 1973 and 1978 indices. The change in the SSS during the 10 year follow up was also used in the analyses.

The scores were classified into three classes with class limits dividing the distrubutions of the scores into thirds $(1=$ low, $\ldots, 3=$ high $)$, separately in each sex/ occupational class group.

\section{Musculoskeletal morbidity}

Musculoskeletal morbidity was assessed as follows.

Rheumatic symptoms-In the questionnaire an inquiry was made about aches and pains in different parts of the body: "Have you suffered from aching, stiffness, tenderness or pain in joints or muscles listed below and how often during the past year?" The list included the following items:

Shoulder, upper arm
Elbow, forearm
Wrist, hand, fingers
Hip joint
Thigh
Knee
Calf
Ankle, foot

$$
\begin{aligned}
& \text { Sole, toes } \\
& \text { Neck, cervical and occipital } \\
& \text { region } \\
& \text { Radiation of cervical pain to } \\
& \text { the arm } \\
& \text { Thoracic region } \\
& \text { Radiation of lumbosacral } \\
& \text { pain to the leg }
\end{aligned}
$$

Each item was scored from 0 (never) to 3 (often or continuously). The left and right side were scored separately. The rheumatic symptoms score is a sum score of all the above items. A mean of the 1973 and 1978 scores was calculated and labelled as the mean rheumatic symptoms score.

Clinical findings-Two trained physiotherapists performed the clinical examination of the musculoskeletal system at each stage of the study. In 1978 the youngest age group was not examined. Pains in the muscles and joints were assessed by palpation, and restrictions in the movements of joints were measured. Ranges of movement in joints were generally measured in active motion, except where this was not feasible (hips, ankles). The guidelines published by the American Association of Orthopedic Surgeons ${ }^{8}$ were used as follows:

$0=$ Symptomless

$1=$ Pain in the movement of the joint and/or on palpation

$2=$ The range of movement restricted by less than 25 per cent of the normal range

$3=$ Restriction by 25 to 50 per cent

$4=$ Restriction by more than 50 per cent

Cervical spine:

$4=$ Restriction by more than 60 degrees and positive compression sign or foramen intervertebralis sign 
Lumbosacral spine:

$4=$ Marked inflexibility or neurological signs in lower extremities (positive Laseque's or Ely's test)

Myalgias in the extremities were scored from 0 (none) to 2 (bilateral).

Twenty one variables, based on the examination, were summed to form the musculoskeletal findings score ( 80 grades). The label "mean musculoskeletal findings score" refers to the mean of the 1973 and 1978 scores.

The scores of musculoskeletal morbidity were classified separately in each sex/occupational class group using class limits that divided the distributions into thirds.

The reliability of the morbidity scores-There was no time trend during the examination period in the means of the musculoskeletal findings score, either at baseline (initial findings) or at the end of the follow up, when calculations were made by two week periods for the total duration of the health examinations. At baseline there were no significant differences between the physiotherapists in the mean scores, similarly calculated by two week periods, but both at the 5 year follow up and at the 10 year follow up such differences were shown. At the follow up examinations, however, the percentage distributions of the examinees by age, sex, and occupational status in the groups assessed by each physiotherapist were very similar. Thus, there should be no systematic error in our results resulting from different sensitivity of the examiners.

Fifty four persons at baseline went through a second examination by the same physiotherapist a short time (up to two weeks) after the first examination. The correlation of the scores was 0.93 in the men and 0.77 in the women.

In 197853 persons and in 198354 persons went through a second examination directly after the first one, made independently by the other physiotherapist. The kappa coefficients of some items (in 1978/1983) were as follows: $0 \cdot 70 / 0.64$ (left humero-scapular joint); $0.76 / 0.61$ (cervical spine); $0.79 / 0.55$ (myalgia of upper arm); and $0 \cdot 74 / 0 \cdot 40$ (lumbosacral spine).

Chronic musculoskeletal diseases-The dichotomous variable "chronic musculoskeletal disease" was based on the questionnaire item: "Have you at present any permanent illness or impairment?" When there was a positive response, the person was asked to write down the illness(es). In the health examination a physician confirmed, mainly by interview, the presence of chronic musculoskeletal diseases. The 10 year incidence of chronic musculoskeletal disease was calculated in those persons who had no such disease at baseline, and the incidence during 1978-83 in those who had no disease at baseline or in 1978 .
LOSS TO FOLLOW UP

Seventy four per cent of the women in the original sample, and $63 \%$ of the men, took part in both re-examinations (table 1). Forty two persons died during the 10 year follow up period. In women, those lost to follow up were younger than those who participated in the re-examinations. In men there were no statistically significant differences in the age distribution of the participants and non-participants. In 1983, half of the sample were still employed by Valmet and a fourth had retired from work.

Table 1 Number of subjects in the sample at baseline, and of subjects who participated in all three examinations

\begin{tabular}{llll}
\hline & & 1973 & 1973,1978 and 1983 \\
\hline \multirow{2}{*}{ Women } & White collar & 144 & 111 \\
& Blue collar & 149 & 107 \\
\multirow{2}{*}{ Men } & White collar & 253 & 154 \\
& Blue collar & 356 & 232 \\
\multirow{2}{*}{ Total } & & 902 & 604 \\
\hline
\end{tabular}

\section{STATISTICAL METHODS}

The significance of the differences in morbidity by sex and occupational class was determined by $t$ tests, which were also used in comparing the mean changes in the morbidity scores by the type of change (increase, decrease) in the stress symptoms score. The associations of the variables with age were assessed using Spearman rank correlation coefficients. Analysis of variance and of covariance (Manova, SPSS-X) were used to assess the associations between the determinant and outcome of interest, and to control for the effect of age. In the longitudinal analyses the baseline level of the outcome variable was also adjusted for by analysis of covariance. The prevalence and incidence rates of chronic musculoskeletal disease were obtained from analyses of variance/covariance by interpreting the class means as proportions.

\section{Results}

The means and standard errors at baseline of the stress symptoms score and the measures of musculoskeletal morbidity by sex and occupational class are presented in fig 1 . The means of both the stress symptoms score and the morbidity scores were higher in the blue collar than in the white collar groups, and higher in women than in men.

Musculoskeletal morbidity increased with age according to all indices. The stress symptons score was significantly associated with age only in men. The change in the SSS during the 10 year follow up was not 
Stress symptoms score

Women Men

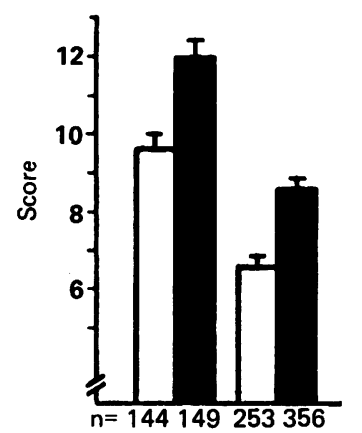

Rheumatic symptoms

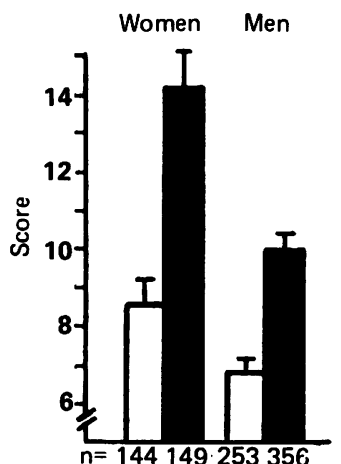

Musculoskeletal findings

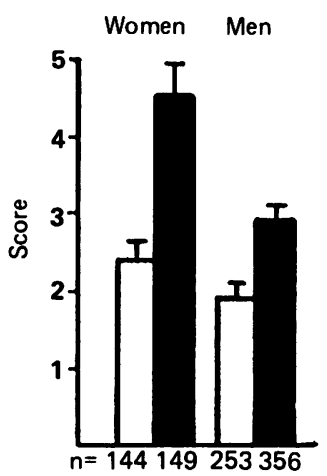

Chronic musculoskeletal disease

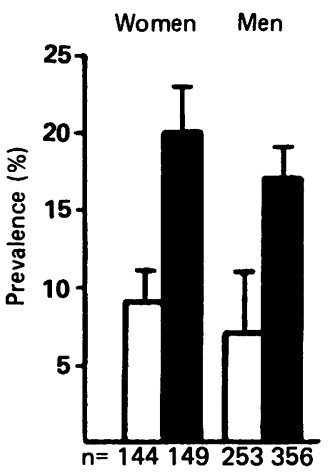

Fig 1 The stress symptoms, rheumatic symptoms, and musculoskeletal findings scores, and the prevalence of chronic musculoskeletal disease at baseline, by sex and occupational class. Columns are means, bars $=S E M$. Statistical analysis (t test): (1) Stress symptoms score: by occupational class, $\mathrm{t}=4.53, p<0.001 ;$ by sex, $\mathrm{t}=7.03, p<0.001$, (2) Rheumatic symptoms: by occupational class, $\mathrm{t}=6 \cdot 04, p<0 \cdot 001$; by sex, $\mathrm{t}=3 \cdot 74, p<0 \cdot 001$, (3) Musculoskeletal findings: by occupational class $\mathrm{t}=5 \cdot 27$, $p<0.001$; by sex, $\mathrm{t}=3.41, p<0.001$, (4) Chronic musculoskeletal disease: by occupational class, $\mathrm{t}=4 \cdot 84, p<0.001 ;$ by sex, $\mathrm{t}=0.55, N S$.

dependent on age in any subgroup. Thus age was taken into the analyses for adjustment in the male groups in all analyses except those of the score changes.

The association of the stress symptoms score with morbidity at baseline

An association was observed between the stress symptoms score on one hand and the rheumatic

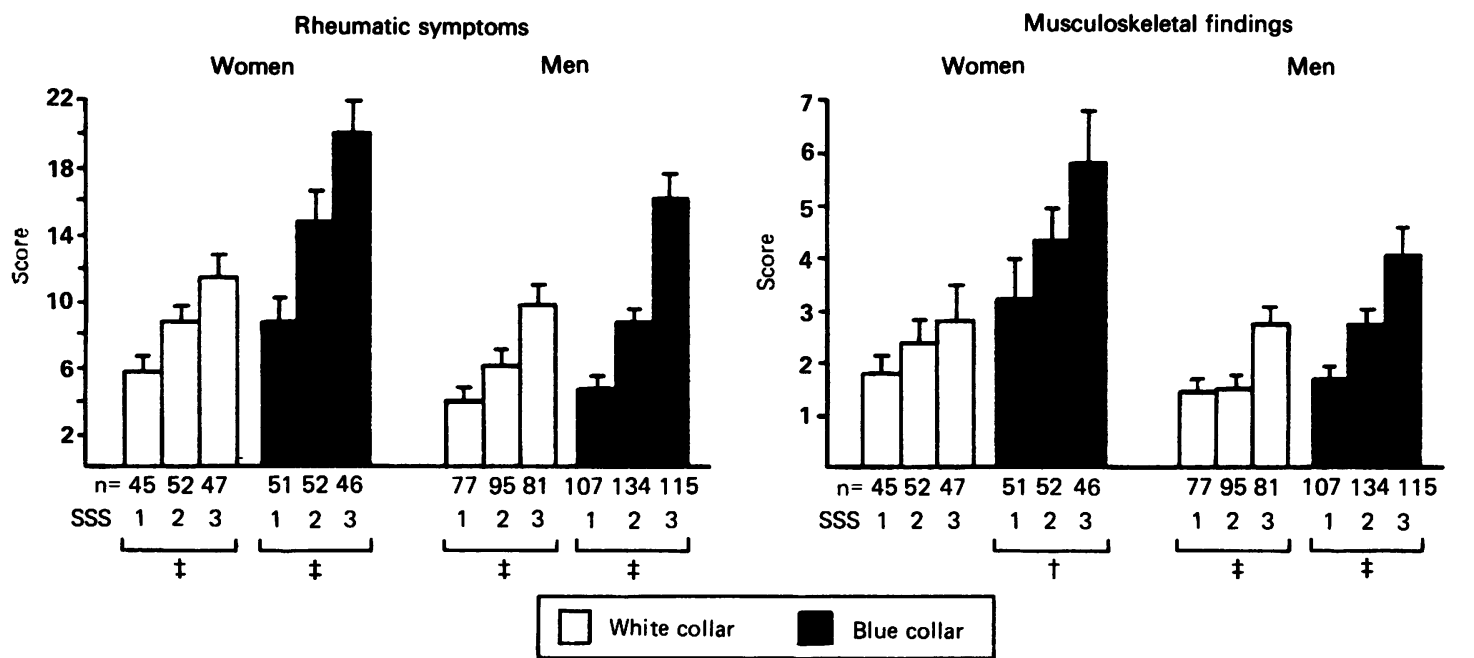

Fig 2 The rheumatic symptoms and musculoskeletal findings scores in three classes of the stress symptoms score (SSS) at baseline, by sex and occupational class. Age adjusted means in men. Columns are means, bars $=S E M$.

$\dagger p<0.01 ; \quad \mp p<0.001$. symptoms score and the musculoskeletal findings score on the other at baseline (fig 2). The SSS was associated with the prevalence of chronic musculoskeletal disease in all groups except the white collar women (fig 4a).

The association of the change in the stress symptoms score with the change in the morbidity scores The change in the stress symptoms score during the 10 
Table 2 Changes in the rheumatic symptoms and the musculoskeletal findings scores during the 10 year follow up, according to whether there was a decrease or an increase in stress symptoms score (SSS) (SSS $\angle 0=$ decrease, $S S S \geqslant 0=$ increase), by sex and occupational class. Data are means $(n)$

\begin{tabular}{|c|c|c|c|c|c|c|}
\hline & \multicolumn{3}{|c|}{ Change in rheumatic symptoms } & \multicolumn{3}{|c|}{ Change in musculoskeletal findings } \\
\hline & $\begin{array}{l}\text { Change in } \\
S S S<0\end{array}$ & $\begin{array}{l}\text { Change in } \\
S S S \geqslant 0\end{array}$ & $t$ & $\begin{array}{l}\text { Change in } \\
S S S<0\end{array}$ & $\begin{array}{l}\text { Change in } \\
S S S \geqslant 0\end{array}$ & $t$ \\
\hline \multicolumn{7}{|l|}{ Women } \\
\hline White collar & $\begin{array}{l}0.25 \\
(63)\end{array}$ & $\begin{array}{l}4 \cdot 31 \\
(48)\end{array}$ & $-2.91 t$ & $\begin{array}{l}5.06 \\
(63)\end{array}$ & $\begin{array}{l}5 \cdot 13 \\
(48)\end{array}$ & -0.06 \\
\hline Blue collar & $\begin{array}{l}1 \cdot 58 \\
(59)\end{array}$ & $\begin{array}{l}5 \cdot 41 \\
(46)\end{array}$ & -1.49 & $\begin{array}{l}5 \cdot 76 \\
(59)\end{array}$ & $\begin{array}{l}5.85 \\
(46)\end{array}$ & -0.07 \\
\hline \multicolumn{7}{|l|}{ Men } \\
\hline White collar & $\begin{array}{r}-0.57 \\
(82)\end{array}$ & $\begin{array}{l}2.07 \\
(71)\end{array}$ & $-2 \cdot 20^{*}$ & $\begin{array}{l}5 \cdot 39 \\
(82)\end{array}$ & $\begin{array}{l}5 \cdot 48 \\
(71)\end{array}$ & $-0 \cdot 10$ \\
\hline Blue collar & $\begin{array}{r}-0.72 \\
(132)\end{array}$ & $\begin{array}{l}4 \cdot 22 \\
(100)\end{array}$ & $-4.04 \ddagger$ & $\begin{array}{l}6.91 \\
(132)\end{array}$ & $\begin{array}{l}6 \cdot 77 \\
(100)\end{array}$ & $0 \cdot 18$ \\
\hline
\end{tabular}

$* \mathrm{p}<0.05 ; \quad+\mathrm{p}<0.01 ; \quad \mp \mathrm{p}<0.001$

year follow up was classified into two classes (decrease, increase), and the mean changes of the morbidity scores in these classes were compared. The class with an increase in the SSS also had an increase in the rheumatic symptoms score, while a decrease of the SSS was associated either with a smaller increase or with a decrease in this score (table 2). No such associations were found with the changes in the musculoskeletal findings score.

While no inferences on causality can be made from

Rheumatic symptoms 1983

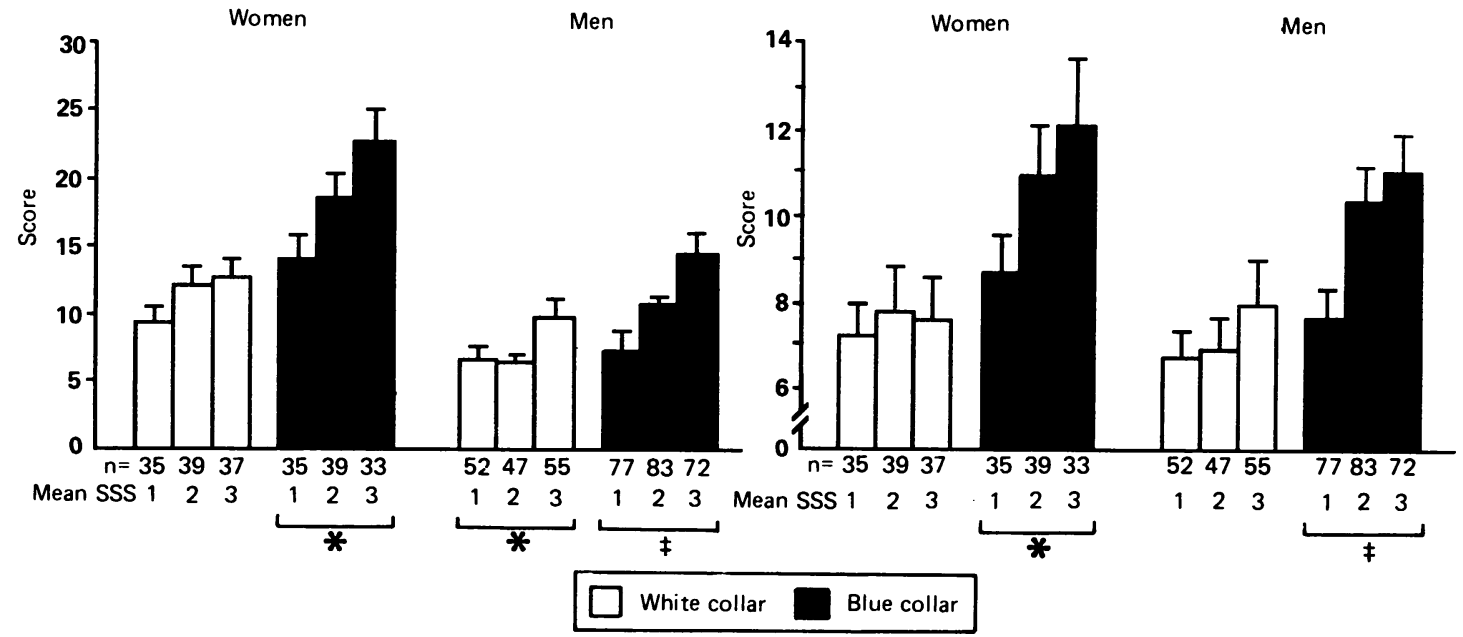

Fig 3 The 1983 rheumatic symptoms and musculoskeletal findings scores, adjusted for the respective baseline scores, in the three classes of the mean stress symptoms scores (SSS) of 1973 and 1978, by sex and occupational class. Age adjusted means in men. Columns are means, bars $=S E M$.

* $p<0.05 ; \quad \pm p<0.001$.

associations observed at one point in time, or from associations of simultaneous changes, two sets of further analyses were made: (1) to test the assumption that stress predicts musculoskeletal morbidity (assumption No 1); (2) to test the assumption that musculoskeletal morbidity produces stress (assumption No 2).

Analyses based on assumption No 1-The mean stress symptoms score predicted the 1983 rheumatic symptoms and musculoskeletal findings scores, when

Musculoskeletal findings 1983

Men 
(a) Baseline

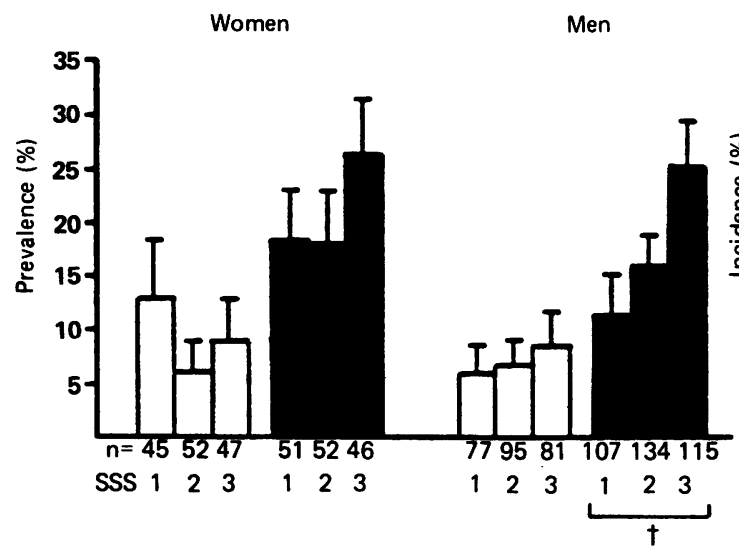

(b) Follow up

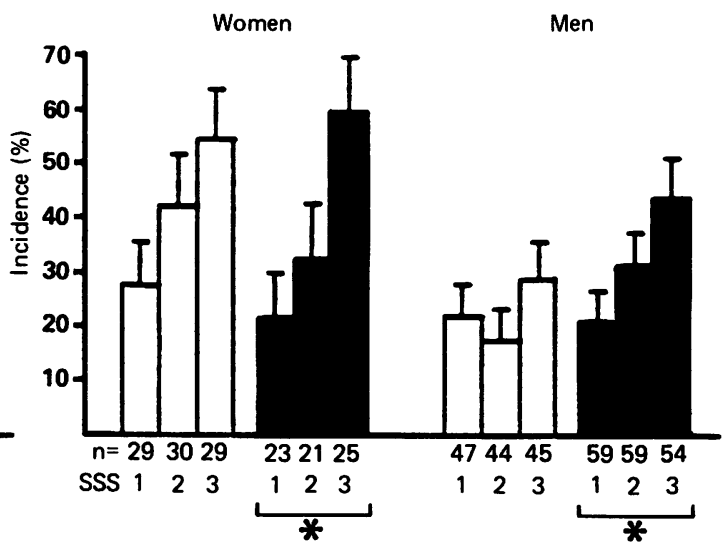

White collar $\square$ Blue collar

Fig 4 (a) Prevalence of chronic musculoskeletal disease in the three classes of the stress symptoms score (SSS) at baseline, and $\dot{\omega}$ (b) the 5 year incidence (1978-83) of chronic musculoskeletal disease in the three classes of the mean SSS of 1973 and 1978 . Ageo 0 adjusted means in men. Columns are means, bars $=S E M$.

$* p<0.05 ; \quad+p<0.01$.

the baseline level of the relevant morbidity score was adjusted for (fig 3). The association of the mean SSS with the 5 year incidence (1978-1983) of chronic musculoskeletal disease is shown in fig $4 \mathrm{~b}$.

Analyses based on assumption No 2-The association of the mean rheumatic symptoms scores of

Stress symptoms 1983

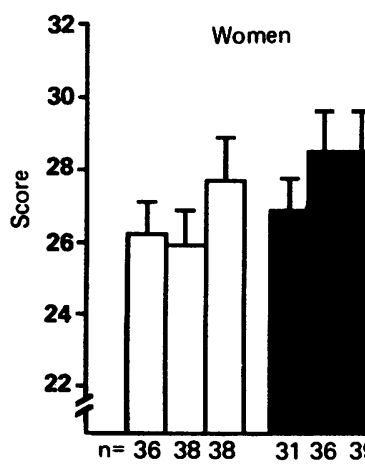

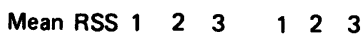

1973 and 1978 with the stress symptoms score at the end of the 10 year follow up is shown in fig 5 . The mean musculoskeletal findings score predicted the 1983 SS\$O in men (fig 5). In both analyses, the 1973 SSS was allowed for.

There was an average decrease in the stress $\overrightarrow{0}$ Stress symptoms 1983

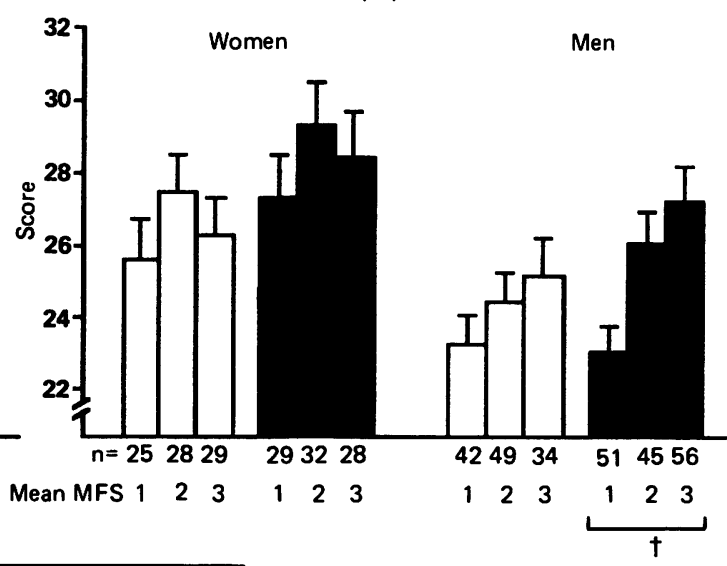

Blue collar

Fig 5 The 1983 stress symptoms score (SSS) adjusted for the baseline SSS, in the three classes of the mean rheumatic symptoms score (Mean RSS) and the mean musculoskeletal findings score (Mean MFS) of 1973 and 1978, by sex and occupational class. Age adjusted means in men. Columns are means, bars $=S E M$. $\dagger p<0.01 ; \quad \mp p<0.001$. 


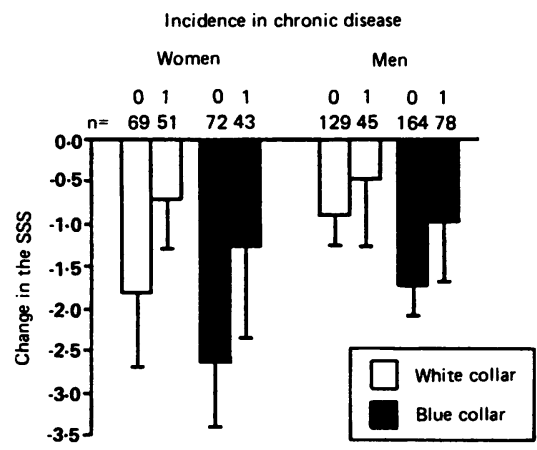

Fig 6 The change in the stess symptoms score (SSS) by the incidence of chronic musculoskeletal disease $(0=$ no disease, $I=$ disease) during the 10 year follow up, by sex and occupational class. Columns are means, bars $=S E M$.

symptoms score during the 10 year follow up in the cohort. The decrease in those who had contracted a chronic musculoskeletal disease was smaller than in those who remained without such a diagnosis (fig 6). None of the differences was statistically significant.

\section{Discussion}

I have shown a relationship to exist between symptoms of stress and musculoskeletal morbidity, the latter measured as rheumatic symptoms, clinical findings, and diagnoses of chronic diseases. Two assumptions about causation were proposed and studied in a longitudinal study design. Measurements were made three times at 5 year intervals.

The possibility that stress is a cause of musculoskeletal disorders was supported by the following evidence: the mean stress symptoms score on the first two occasions predicted (a) the level of the morbidity scores at the end of the follow up, allowing for the level of the relevant morbidity score at baseline, and (b) the incidence of chronic muculoskeletal diseases during the second 5 year period.

The possibility that musculoskeletal disorders produce stress was also supported by the results, though somewhat less consistently than the first assumption.

The interpretation of the evidence supporting the second assumption is straightforward. Degeneration of the locomotor system and the associated symptoms interfere with movement and cause difficulties in pursuing everyday tasks, etc. They are, therefore, sources of stress.

As to the interpretation of the evidence for the first assumption, some observations on the implements of measurement are necessary. The indicator of stress was based on the abundance of various symptoms perceived by the subjects. Many of the items were similar to those in the instruments originally designed for the screening for psychiatric disorder. ${ }^{910}$ Formulations such as "psychosomatic symptoms" and "symptoms of stress" have been widely used when referring to these symptoms. ${ }^{11} 12$ It has also been suggested that they may more properly be seen to reflect states of deprivation. ${ }^{13}$ The possibility that some of the items in the stress symptoms score would be symptoms of somatic diseases has been considered in our study population ${ }^{6}$ and found unlikely. That symptoms of musculoskeletal derangement would be included in the stress symptoms score is also unlikely, with the possible exception of headache, which may be associated with neck-shoulder problems.

The probability that a chronic musculoskeletal disease will be diagnosed may be enhanced under stress because of an added readiness to consult a doctor. ${ }^{6}$ To obtain a more objective measure of the state of the locomotor system a clinical examination by a physiotherapist was made. There is a subjective element embedded even in this score: for example, whether and how much pain a person feels or reports during palpation in an examination may be dependent on the level of stress she/he is subject to. The absence of any association between the change in the stress symptoms and the change in the findings suggests that little or none of this effect is operative in the musculoskeletal findings score.

The sensitivity of the investigator also influences the findings. An attempt at enhancing the reproducibility of the method was made by ensuring that the same person was the main physiotherapist at all stages of the study. In spite of this, there was a systematic increase of findings in groups that were of the same age in 1973, 1978 and 1983. A reason for the increased sensitivity of the method may be the added working experience gathered by the physiotherapist during the follow up period. However, this does not affect my conclusion.

My results lend credence to the view that stress increases the occurrence of musculoskeletal disorders. Some of the effect may be mediated by influences on help seeking behaviour, but the results also suggest that stress may produce changes in the physical state of the musculoskeletal system.

This study was supported by a grant from the Medical Research Council of the Academy of Finland.

\section{References}

${ }^{1}$ Anderson KO, Bradley LA, Young LD, McDaniel LK, Wise CM. Rheumatoid arthritis: review of psyshological factors related to etiology, effects and treatment. Psychol Bull 1986; 98: 358-87.

${ }^{2}$ Lichtenberg PA, Skehan MW, Swensen $\mathbf{C H}$. The role of personality, recent life stress and arthritic severity in predicting pain. J Psychosom Res 1984; 28: 231-6. 
${ }^{3}$ Lichtenberg PA, Swensen CH, Skehan MW. Further investigation of the role of personality, lifestyle and arthritic severity in predicting pain. $J$ Psychosom Res 1986; 30: 327-37.

${ }^{4}$ Lunghi M, Miller P, McQuillan W. Psychosocial factors in osteoarthritis of the hip. J Psychosom Res 1978; 22: 57-63.

${ }^{5}$ Leavitt F, Garron DC, Bieliauskas LA. Psychological disturbance and life event differences among patients with low back pain. J Consult Clin Psychol 1980; 48: 115-6.

${ }^{6}$ Aro S. Stress, morbidity, and health-related behaviour. A five-year follow-up study among metal industry employees. Scand J Soc Med 1981; 9, suppl 25.

${ }^{7}$ Kalimo R. Stress in work. Scand J Work Environ Health 1980 ; 6, suppl 3.

${ }^{8}$ American Association of Orthopedic Surgeons. Joint motion-method of measuring and recording. Chicago: AAOS, 1965.

9 MacMillan AM. The Health Opinion Survey: a technique for estimating prevalence of psychoneurotic and related types of disorder in communities. Psychol Rep 1957; 3: 325-39.

${ }^{10}$ Langner TS. A twenty-two item screening scale of psychiatric symptoms indicating impairment. $J$ Health Human Behav 1962; 3: 269-76.

11 Seiler LH. The 22-item scale used in field studies of mental illness: a question of method, a question of substance, and a question of theory. J Health Soc Behav 1973; 14: 252-64.

12 Wheaton B. Uses and abuses of the Langner Index: a reexamination of the findings on psychological and psychophysiological distress. In: Mechanic D, ed. Symptoms, illness behaviour, and help-seeking. New York: Prodist, 1982: 25-53.

${ }^{13}$ Hasan J. Way-of-life, stress, and differences in morbidity between occupational classes. In: Fox AJ, ed. Health inequalities in European countries. Aldershot: Gower Press, 1989: 372-85. 